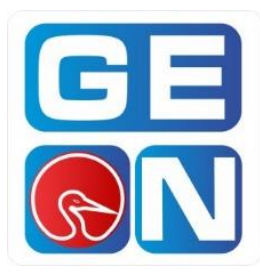

Revista GEON (Gestión, Organizaciones y Negocios.) ISSN: 2346-3910 en línea

revistageon@unillanos.edu.co

Universidad de los Llanos

Colombia

Moreno Cortés, Karla Coréi; Morales Espíndola, María

Guadalupe; Romano Cadena, María Martha del Socorro ${ }^{\text {ii }}$.

Modelo de capacitación para trabajadores de empresas

de servicios en el contexto actual

Revista GEON, Vol. 5, No. 2, 2018

Pág. 24-37

Disponible en: $\underline{\text { https://doi.org/10.22579/23463910.26 }}$

' https://orcid.org/0000-0001-5673-2428

ii https://orcid.org/0000-0002-2402-0933

Esta publicación se encuentra bajo licencia: Creative Commons

ReconocimientoNoComercial-

SinObraDerivada 4.0 Internacional

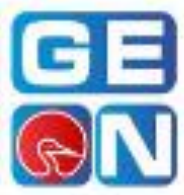

Los nuevos retos administrativos del siglo XXI, obligan a indagar e implementar nuevas estrategias de capacitación para impactar en el desempeño y la competitividad laboral que exigen cada vez más los clientes 99

RevistaGEON

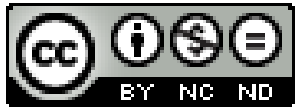




\section{Modelo de capacitación para trabajadores de empresas de servicios en el contexto actual}

\section{Training model for workers of service companies in the current context}

\section{María Guadalupe Morales Espíndola \\ gualumespindola@hotmail.com}

Administradora Pública y Dra. en Investigación educativa, Benemérita Universidad Autónoma de Puebla BUAP, México.

\section{Karla Coré Moreno Cortes \\ karla.morenoco@correo.buap.mx}

Lic. Administración de Hoteles y Restaurantes y Dra. en Investigación educativa, Benemérita Universidad Autónoma de Puebla BUAP, México

\section{María Martha del Socorro Romano Cadena Martha.romano@correo.buap.mx}

Administradora de Empresas y Dra. en Investigación Educativa, Benemérita Universidad Autónoma de Puebla BUAP, México.

Revista GEON Vol. 5 No 2 julio-diciembre 2018 ISSN 2346-3910 en línea http://revistageon.unillanos.edu.co/index.php/geon/article/view/26 


\title{
Resumen
}

El presente trabajo tiene como objetivo analizar teórica y prácticamente la razón por la cual la capacitación representa en la actualidad una inversión de corto y mediano plazo que enriquece el capital humano en las organizaciones de la sociedad del conocimiento del siglo XXI. Se propone una metodología de impacto para empresas de servicios como las del Sector Salud, cuyas condiciones de trabajo (al igual que otros servicios) no siempre favorecen o garantizan una capacitación exitosa.

El capacitador debe enfrentar las actitudes negativas de las personas, quienes se presentan desmotivadas, con la tendencia de culpar al nivel directivo de las situaciones adversas, con una falta de disposición a la capacitación, con rechazo por tener que realizar este proceso a contra turno o utilizando un $50 \%$ de su tiempo libre. Son circunstancias que impiden, en la mayoría de los casos, llegar al cumplimiento de los objetivos programados.

En nuestro país, existe la obligatoriedad legal, dada por la Constitución Política y por la Ley Federal del Trabajo, de aplicar Programas de Capacitación. Esto con el fin de proteger al trabajador de un despido por inadecuado desempeño, causado por la falta de capacitación y actualización en su trabajo. No siempre se tiene plena consciencia de ello.

Palabras claves: Capacitación, capital humano, Empresa de servicios

\begin{abstract}
The objective of this work is to analyze theoretically and practically the reason of: why training represents an investment of short and medium term who enriches human capital in organizations of the knowledge society of the XXI century? impact methodology for service companies such as the health sector, whose working conditions (like other services) do not always favor or guarantee a successful training is proposed.

The trainer must face the negative attitudes of people who are unmotivated, with a strong tendency to blame the management level of adverse situations, with an unwillingness to training, with rejection by having to perform this process against shift or with $50 \%$ of their free time. They are circumstances that prevent, in most cases, come to meeting program objectives.

In our country, a legal obligation is given by the Constitution and the Federal Labor Law, for implementing training programs. In order to protect workers from dismissal for poor performance, caused by lack of training
\end{abstract}


and updating their work. In fact, workers have not always been fully aware of that.

Keywords: Training, Human Capital, Business Services

\section{Introducción}

El Talento Humano representa el factor más importante que posee una organización y del cual depende la eficiencia con la que se realizan sus procesos y, por ende, el cumplimiento de sus principales objetivos.

La capacitación es una actividad continua y permanente enfocada a desarrollar el potencial del capital humano a través de la habilitación y actualización de conocimientos y herramientas necesarias para generar nuevas competencias, cambios de actitud y aptitud para su mejor desempeño en la organización.

Sin embargo, en el marco de las necesidades del siglo XXI, en las cuales los trabajadores tienen varios empleos, trabajan horas extra y cada vez rinde menos el salario; dedicar tiempo para la actualización, es una tarea complicada. Aunado a lo anterior es importante verificar ¿cuáles son las mejores opciones de capacitación?, para ello deben analizarse tanto las experiencias buenas como las malas, ya que el fracaso es únicamente una etapa en el camino al éxito. (Pérez Paredes, Torralba Flores, \& D., 2016)

Aunado a lo anterior, la inmersión en nuevas tecnologías provoca distractores que antes no existían; los empleados están al pendiente de sus teléfonos celulares y es cada vez más difícil lograr la concentración.

La capacitación es en la actualidad un proceso legalmente obligado para los empresarios y debe planearse de forma correcta. Aplicada adecuadamente, genera una mayor productividad y competitividad en las organizaciones.

Por su parte, los clientes o usuarios son cada día más exigentes, pues existen empresas que están en constante competencia y buscan lograr la satisfacción de sus necesidades de la mejor manera.

Actualmente, el mercado está más orientado a servicios y no a productos, por lo que el éxito de la empresa depende del conocimiento, habilidades y destrezas que tengan los colaboradores. Cuando el trabajo de los empleados es valioso y organizado, las empresas pueden alcanzar ventajas competitivas. (Ruiz, 2016)

Es por lo anterior que, con programas de capacitación bien estructurados, que estén basados en procesos de detección de necesidades y realizados con las metodologías adecuadas, se pueden conocer las áreas de oportunidad de cada trabajador. De igual manera, mediante las sesiones pertinentes, es posible, mejorar su desempeño y la comunicación interpersonal, logrando con ello una 
integración mucho más efectiva en la búsqueda de los objetivos empresariales.

La obsolescencia de las máquinas y equipos se detecta y se corrige a través de las acciones de mantenimiento, sustitución o actualización, por tanto, la obsolescencia del factor humano también debe evitarse monitoreando de manera permanente el desempeño y la necesidad de adquirir nuevos conocimientos, técnicas y habilidades, que contribuyan a una mayor productividad y a una actitud más positiva y colaboradora. La tecnología no sirve de mucho si no existe el elemento humano que agregue valor a los procesos de producción o de servicios. (Macías, 2016)

El presente artículo se conforma de un marco teórico conceptual, en donde se revisan las leyes referentes a capacitación, así como las teorías que fundamentan el estudio. Posteriormente, se continúa con descripción de la metodología utilizada en la investigación, aterrizada al caso de estudio del personal de una Unidad del Instituto Mexicano Del Seguro Social (IMSSS), subsecuentemente se detallan los resultados de la investigación y se cierra el artículo con conclusiones y recomendaciones pertinentes.

\section{Contexto teórico y conceptual}

El proceso para proporcionar competencias para un trabajo, se denomina capacitación, en cambio el proceso para acentuar o adquirir valores, estilos, trabajo en equipo y otras facetas de la personalidad, se denomina desarrollo (Arias \& Heredia, 2006).
La obligación de capacitación y adiestramiento se encuentra regulada en el último párrafo del apartado $\mathrm{A}$, del artículo 123 de la Constitución Política de los Estados Unidos Mexicanos, donde textualmente dice: "También será competencia exclusiva de las autoridades federales la aplicación de las disposiciones de trabajo [...] y respecto a las obligaciones de los patrones en materia de capacitación y adiestramiento de sus trabajadores [...]" (Constitución Política de los Estados Unidos Mexicanos., 2017)

Por su parte, la Ley Federal del Trabajo también regula esta obligación patronal en el capítulo III bis, al referirse a la capacitación y adiestramiento de los trabajadores en los artículos del 153-A al 153-X, marcando una serie de requisitos, tanto de los patrones como de los trabajadores y los capacitadores. Estos últimos deberán estar respaldados por una preparación profesional en el área que se pretenda impartir, además debe acreditar ante la Secretaría de Trabajo y Previsión Social no estar ligados a instituciones o personas que propaguen algún credo religioso y la autorización otorgada puede revocarse si se contraviene alguna de las obligaciones anteriores. (Ley Federal del Trabajo, 2016)

La capacitación es un proceso educacional formal que se imparte de manera organizada y sistemática, en la que los participantes adquieren y desarrollan conocimientos y habilidades relativas a su trabajo. Tiende a modificar actitudes personales y vicios laborales. De igual manera, conlleva la intención de integrar al trabajador a su puesto de trabajo, la organización, el mantenimiento e 
incremento de su eficiencia laboral, que finalmente redundará en el progreso personal y de la empresa. (Rodriguez \& Morales, 2008).

En el año 2014, la empresa Deloitte Consulting LLP y Bersin por Deloitte, realizó un estudio; a través de una encuesta que incluyó a 2.532 empresas y líderes de recursos humanos en 94 países. La encuesta cubrió las industrias más importantes y todas las situaciones geográficas del mundo. Nuestra meta fue lograr un mayor entendimiento de las prioridades y estado de preparación de los ejecutivos y profesionales de RR. HH. alrededor del mundo, aportando conocimiento en relación a las acciones que pueden tomar los líderes para desarrollar el talento y la agenda de RR. $\mathrm{HH}$.

En él, se concluyó que el hecho de hacer más no es suficiente. Hoy en día las organizaciones tienen que gestionar a su gente de una manera distinta, generando el imperativo de innovar, transformarse y rediseñar las prácticas de capital humano.

El capital humano es un concepto que remite a la productividad de los trabajadores en función de su formación y experiencia de trabajo. El mismo busca dar cuenta de distintas ventajas en términos de generación de valor considerando al aporte humano que se realiza en un mercado determinado. Por extensión, muchas veces se utiliza el término "capital humano" para dar cuenta de los recursos humanos que tiene una empresa, de sus competencias conjugadas que derivan en una mejora general en la producción. De alguna manera puede decirse que el concepto de capital humano remite al viejo concepto del trabajo como factor productivo, poniendo esta vez el énfasis en la formación de ese factor productivo. (Definición mx, 2016)

Tabla 1. Tres áreas clave de enfoque estratégico

\begin{tabular}{|c|c|c|}
\hline $\begin{array}{l}\text { Liderar y } \\
\text { desarrollar }\end{array}$ & $\begin{array}{l}\text { Atraer e } \\
\text { involucrar }\end{array}$ & $\begin{array}{l}\text { Transformar y } \\
\text { reinventar }\end{array}$ \\
\hline $\begin{array}{l}\text { Líderes en } \\
\text { todos los } \\
\text { niveles: } \\
\text { disminuir la } \\
\text { brecha entre } \\
\text { las } \\
\text { expectativas y } \\
\text { la disposición } \\
\text { real }\end{array}$ & $\begin{array}{l}\text { Reconsiderar } \\
\text { la adquisición } \\
\text { de talento: } \\
\text { desarrollo de } \\
\text { nuevos } \\
\text { enfoques para } \\
\text { el nuevo } \\
\text { contexto }\end{array}$ & $\begin{array}{l}\text { Entrenamiento } \\
\text { del equipo de } \\
\text { RRHH: } \\
\text { transformar a } \\
\text { los } \\
\text { profesionales de } \\
\text { RRHH en } \\
\text { consultores de } \\
\text { negocios } \\
\text { calificados }\end{array}$ \\
\hline $\begin{array}{l}\text { Redefinir el } \\
\text { aprendizaje } \\
\text { corporativo: } \\
\text { prepárese } \\
\text { para una } \\
\text { revolución }\end{array}$ & $\begin{array}{l}\text { Más allá de la } \\
\text { retención: } \\
\text { generar pasión } \\
\text { y propósito }\end{array}$ & $\begin{array}{l}\text { Analítica del } \\
\text { talento en } \\
\text { práctica: de la } \\
\text { conversación al } \\
\text { aporte de Big } \\
\text { Data }\end{array}$ \\
\hline $\begin{array}{l}\text { La gestión del } \\
\text { desempeño ya } \\
\text { no funciona: } \\
\text { reemplace los } \\
\text { rankings por } \\
\text { la } \\
\text { capacitación y } \\
\text { el desarrollo }\end{array}$ & $\begin{array}{l}\text { De la } \\
\text { diversidad a la } \\
\text { inclusión: } \\
\text { apartarse de la } \\
\text { conformidad } \\
\text { hacia la } \\
\text { diversidad } \\
\text { como } \\
\text { estrategia de } \\
\text { negocios }\end{array}$ & $\begin{array}{l}\text { Carrera hacia la } \\
\text { tecnología en la } \\
\text { nube: integrar el } \\
\text { talento, los } \\
\text { RR.HH. y las } \\
\text { tecnologías del } \\
\text { negocio }\end{array}$ \\
\hline $\begin{array}{l}\text { La búsqueda } \\
\text { de } \\
\text { capacidades } \\
\text { en la fuerza } \\
\text { laboral: crear } \\
\text { una cadena } \\
\text { global de } \\
\text { suministro de } \\
\text { habilidades }\end{array}$ & $\begin{array}{l}\text { El empleado } \\
\text { agobiado: } \\
\text { simplificar el } \\
\text { entorno de } \\
\text { trabajo. }\end{array}$ & $\begin{array}{l}\text { La función } \\
\text { global y local } \\
\text { de RR.HH.: } \\
\text { equilibrio entre } \\
\text { la escala y la } \\
\text { agilidad. }\end{array}$ \\
\hline
\end{tabular}

Fuente: Deloitte Consulting LLP (2014)

El informe de Tendencias Globales de Capital Humano, resultado de un extenso estudio global que implicó varios meses de trabajo, brinda orientación y 
recomendaciones en relación a estas importantes estrategias. ( Deloitte Consulting LLP y Bersin por Deloitte, 2014)

Ese año, las doce tendencias fundamentales de Capital Humano se organizaron en tres grandes áreas (ver Tabla 1)

Como se observa en la Tabla 1, la nueva tendencia a seguir para los responsables de R. H. es simplificar los procesos para ayudar a los colaboradores a gestionar el flujo masivo de información en el trabajo, a desarrollar plenamente sus habilidades, y a construir una cultura de colaboración, empoderamiento e innovación.

Empresas de servicios. A este sector pertenecen todas las unidades económicas que ofrecen algún servicio, como: hospitales, escuelas, peluquerías, clubes deportivos, bancos, restaurantes, hoteles, centros de espectáculos, empresas de transportes y de comunicaciones, entre muchas otras. Son una actividad económica terciaria que utiliza productos que provienen de actividades económicas primarias y secundarias.

Este sector incluye a personas que realizan trabajos especializados, como: médicos, enfermeras, abogados, meseros, ingenieros, estilistas, masajistas, entrenadores deportivos, maestros, artistas, choferes, azafatas, etcétera.

En los servicios educativos las mujeres son mayoría. $61.2 \%$ Mujeres y $38.8 \%$ Hombres. Además, diez entidades federativas concentraron $67.7 \%$ del personal ocupado total a nivel nacional. (INEGI, 2015)
El sistema que se usa en México para clasificar la información de las actividades económicas se llama Sistema de Clasificación Industrial de América del Norte (SCIAN). Unifica datos, evita problemas de interpretación y facilita comparativos entre municipios y entidades de México y con los países de Estados Unidos de América y Canadá, así como otras organizaciones de estadística.

El sector se divide en 293 clases de actividad, de acuerdo con esta clasificación las que suman la mayor cantidad de unidades económicas dedicadas a los servicios en México se muestran en la Tabla 2

Tabla 2. Unidades económicas dedicadas a los

Servicios en México

\begin{tabular}{|l|c|}
\hline Clase de actividad & $\begin{array}{c}\text { Unidades } \\
\text { económicas }\end{array}$ \\
\hline $\begin{array}{l}\text { Otros servicios excepto } \\
\text { actividades del gobierno }\end{array}$ & 395014 \\
\hline $\begin{array}{l}\text { Servicios de alojamiento } \\
\text { temporal y de preparación de } \\
\text { alimentos y bebidas }\end{array}$ & 277436 \\
\hline $\begin{array}{l}\text { Servicios de salud y de } \\
\text { asistencia social }\end{array}$ & 102940 \\
\hline
\end{tabular}

Fuente: www.inegi.org.mx/parque_económico/servicios

En México hay 1013743 unidades económicas dedicadas a los servicios y 5 215808 personas que laboran en este sector. (INEGI, 2015)

La economía mexicana tuvo un crecimiento de 2.2 por ciento anual en el segundo trimestre del año 2015, porcentaje al cual el sector servicios aportó 1.8 puntos y tuvo un aumento de 3.1 por ciento anual en este mismo periodo, de acuerdo con datos del INEGI, lo que indica que el sector servicios cada vez está aportando mayores beneficios a la economía del país. 
Las actividades relacionadas con los servicios contribuyeron a la expansión del Producto Interno Bruto (PIB), en el 85 por ciento. (El Financiero, 2015)

En un análisis realizado por la OCDE (Organización para la Cooperación y Desarrollo Económico), en enero de 2015, se menciona que muchas empresas modernas en México emplean trabajadores altamente calificados y con buena formación académica, en particular en las industrias aeroespacial, automovilística, alimentaria y de bebidas. Con todo, hay sectores de la economía que siguen caracterizándose por altos índices de informalidad, trabajo poco calificado, productividad escasa y tecnologías obsoletas. Alrededor de $57 \%$ de los trabajadores tienen empleos informales, mientras que las empresas del ámbito no formal emplean a millones de personas que carecen de acceso a un ingreso estable, buena educación, atención integral de la salud y servicios financieros asequibles, lo que impide la formación de capital humano. (OCDE, 2015).

Lo mencionado permite inferir que los trabajadores de cualquier sector que deseen permanecer durante su vida productiva en una empresa formal deben estar dispuestos a participar en los procesos de capacitación y desarrollo que le son exigidos para el mejor desempeño de sus competencias laborales.

Aunado a ello, los capacitadores que proporcionen sus servicios deben dominar perfectamente los conocimientos, destrezas y habilidades que transmiten, pero, sobre todo, saber identificar $y$ manejar las estrategias de adaptación al tipo de personas a las cuales dirigen su ejercicio para cumplir el objetivo de la empresa que lo contrata y al mismo tiempo, su objetivo educativo y de formación.

Especialmente debe considerarse que el sector servicios presenta características específicas debido a que los "productos" que oferta son intangibles, por ejemplo; un médico proporciona un servicio, pero la salud es algo que no se puede ver, tocar o comprar, solamente se siguen indicaciones $\mathrm{y}$ se toman medicamentos, pero la tangibilidad es inexacta; de igual forma ocurre con los servicios prestados por un abogado o un profesor.

Aunado a lo anterior, los servicios son heterogéneos, es decir, debido a la naturaleza de estos, requieren al factor humano para su entrega y desempeño; las personas no actúan todos los días por igual, no siempre tienen la misma sonrisa o el mismo tono de voz; es decir, se hace un esfuerzo por estandarizar los procesos de servicio, sin embargo, resulta complicado ser siempre iguales, pues el servicio requiere personas, atendiendo personas.

\section{Metodología}

Para realizar el presente trabajo, se utilizó el método de investigación documental, empleando la técnica de sistematización bibliográfica y el instrumento de ficha de trabajo. Se considera una investigación exploratoria y descriptiva, por haber tenido un acercamiento a la realidad en un primer momento con 412 personas de una Unidad del IMSS en Puebla (275 mujeres y 137 
hombres), a quienes se proporcionó una capacitación adaptada a las condiciones de su contexto, lo que sirvió de base para proponer un modelo para empresas de servicios.

Se realizó la evaluación inmediata del curso, sin embargo, para efectos de impacto, se realizará en una segunda etapa, una medición de efectividad de competencias concretas que los participantes aprendieron en dicha capacitación.

La norma de Diseño de Cursos para Adultos en la que se fundamenta esta nueva propuesta se denomina CONOCER (Conocimiento, Competitividad y Crecimiento)

En ella se establece el Estándar de Competencia (EC0217) cuya acreditación permite certificar a quienes aprenden a desarrollar cursos de formación del capital humano presenciales grupales a través de la elaboración de manuales e instrumentos de evaluación. Para ello, legalmente no se requiere que el participante cuente con un título profesional.

El EC0217 se fundamenta en criterios rectores de legalidad, competitividad, libre acceso, respeto, trabajo digno y responsabilidad social.

Se recomienda que se lleve a cabo en el lugar de trabajo y durante la jornada laboral, sin embargo, pudiera realizarse de manera simulada en un área experimental con la infraestructura necesaria para llevar a cabo el desarrollo de todos los criterios de evaluación referidos en el Estándar de Competencia.
Con relación a la competencia sobre el conocimiento de Educación para Adultos, el capacitador debe saber que los principios que rigen su aprendizaje son: Necesidad de saber, Disposición para aprender, Motivación para aprender, Recuperación de la experiencia, Des aprendizaje, Aplicación práctica en la vida real.

Caso práctico. capacitación a personal de un hospital. Para realizar esta investigación, se tomó como muestra a 412 trabajadores del sector salud, es decir de una Unidad Hospitalaria del Instituto Mexicano del Seguro Social (IMSS), ubicado en la Ciudad de Puebla.

Se impartieron 16 cursos con duración de 3 horas durante el mes de julio de 2016. Los horarios fueron de 9 a 12 horas, de 14 a 17 horas y de 20 a 23 horas (con la finalidad de cubrir los 3 turnos en que laboran los trabajadores), Previo al curso, en la etapa de diagnóstico de necesidades de capacitación (la cual forma parte de la metodología propuesta) se identificaron los siguientes problemas específicos:

El personal de la empresa hospitalaria vivió un reciente cambio en la Dirección, por lo cual los trabajadores se encontraban en una etapa de readaptación a las prácticas hospitalarias. Existen reportes de un aumento de quejas por parte de los usuarios del hospital, específicamente, perciben falta de compromiso, amabilidad y calidez del personal; en especial de los médicos.

Al ser un hospital-escuela que recibe médicos internos y residentes de primer a cuarto año; los pacientes perciben que 
“demasiadas personas los revisan y no se sienten con la misma confianza; piensan que se está experimentando con ellos"

Por su parte, el personal se encuentra muy presionado, ya que se adquirió un software para trabajar los expedientes clínicos de forma digital, se cambió el proceso de trabajo al que ya se habían acostumbrado en papel y mencionan que a pesar de que les prometieron que la nueva forma sería más ágil y mejor, existen fallas o caídas del sistema y algunos procesos se volvieron más lentos, esto generó molestia en el personal debido a constantes problemas con el usuario y ellos se sienten reacios al cambio.

Así mismo, tanto el personal directivo, como los médicos y administrativos, deben pasar por un proceso de reacreditación del hospital, por lo cual el 80 por ciento de su personal, debe asistir al menos a 3 sesiones de capacitación, lo que marca una obligatoriedad en la actualización, como se mencionó con anterioridad en el presente artículo. Esta situación genera molestia, ya que los trabajadores mencionan que tienen mucho trabajo y no les agrada una capacitación tradicional; la consideran aburrida y pérdida de tiempo.

En cursos previos, (impartidos por otros capacitadores) se vivieron episodios en los que, participantes discutieron con el facilitador y varios de ellos se salían de los cursos argumentando "emergencias" en su área laboral, es decir no todos los que iniciaban la capacitación, se esperaban hasta el término de esta.

Ante este panorama, se procedió a la etapa de diseño del curso, es importante mencionar, que en esta metodología propuesta, la capacitación debe tener un impacto más allá de la obligatoriedad que por ley, se exige a los empresarios; ya que en el contexto actual se requiere un incremento en la competitividad laboral; especialmente en el sector servicios, debido a que se trabaja con percepciones subjetivas de los usuarios por el alto grado de intangibilidad del "producto" ofertado y en particular en el Sector Salud, los pacientes y familiares de enfermos, se encuentran más sensibles al trato, ya que están preocupados o con dolor, es decir, la capacitación debe verse como elemento para aumentar la competitividad del trabajador.

Así pues, se diseñó el curso denominado: "Taller de Atención al Usuario y Trato Digno", basado en una "metodología de aprendizaje significativo", la cual se define como: el proceso en el que el participante genera conocimiento nuevo, con la ayuda de un facilitador que lo apoye para reconstruir y reajustar información que ya posee debido a sus experiencias previas. Así mismo se utilizaron actividades de aprendizaje constructivista y colaborativo, porque en la etapa de diagnóstico previa al diseño, se determinó que el personal de salud ya tenía los conocimientos necesarios para dar un buen servicio, sin embargo, la actitud era el problema en este caso específico.

El taller se diseñó cumpliendo los lineamientos de los estándares 301 y 217 y de la norma CONOCER; es decir se elaboró una carta descriptiva con los objetivos, requerimientos y timing del curso; así como el material de evaluación, 
la guía del instructor o facilitador y la guía del participante.

\section{Resultados}

El taller se dividió en tres etapas: sensibilización, aplicación y decálogo de acciones específicas.

En la etapa de sensibilización, se les pidió a los participantes que se entrevistaran unos a otros y mencionaran el motivo por el cual habían decidido trabajar en el hospital. Esta actividad no solo tenía el objetivo de servir de rompehielos, se hizo específicamente para que los participantes recordaran sus motivaciones laborales; muchos de ellos describieron su vocación en el área médica, especialmente enfermeras $\mathrm{y}$ médicos y algunos mencionaron su esfuerzo para ser contratados en el hospital; este hecho hizo que revaloraran el tener un empleo en el hospital.
Posteriormente, se procedió a mencionar las causas del mal servicio "imputables a los procesos administrativos, a la mala actitud/aptitud de los empleados y a las brechas de servicio entre lo que el cliente espera, lo que recibe y lo que percibe; estas últimas basadas en el modelo de Parasuraman, Zeithman y Berry (2008)" (Figura 1). Lo anterior se manejó en un tono jovial con el uso de videos que exageraban las fallas e incluían un toque de comedia.

En la etapa de aplicación, se les pidió a los participantes que trabajaran por equipos, en estos grupos, debían mencionar "todos los elementos que intervenían en la satisfacción del usuario y que eran importantes para dar un trato digno al mismo", se les entregó una hoja de rota folio, y se les pidió la dividieran en 2 partes; mencionando en una mitad, lo que estaba en sus manos resolver y en la otra, lo que no.

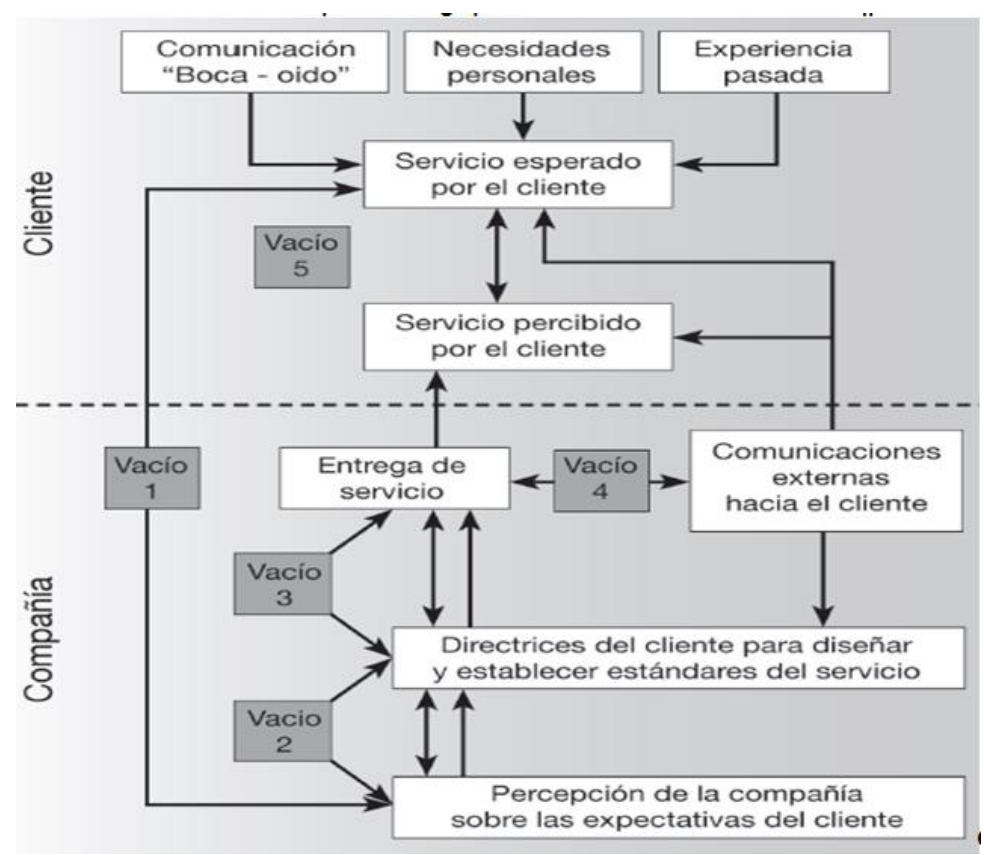

Figura 1. Brechas de Servicio Elaborado por Parasuramann, Zeithaml y Berry (2008) 
Posteriormente debían exponer sus conclusiones al grupo. Esta actividad, tuvo como finalidad que fueran los propios trabajadores los que identificaran, basados en sus experiencias, los elementos clave en los que debían poner atención para dar calidad y calidez al usuario. Se logró un entendimiento interdepartamental, ya que escucharon el punto de vista y la problemática que viven compañeros de archivo, secretarias, administrativos, personal de limpieza, personal médico, personal de vigilancia, enfermeras, camilleros, etc.; sin distinción por su cargo o puesto. Esto los llevó no solo a un consenso grupal, sino que los hizo reflexionar sobre algunas acciones que pueden afectar el trabajo de otros, e incluso se propusieron cambiar procedimientos específicos con la finalidad de trabajar mejor en conjunto.

Como se mencionaba con anterioridad, una constante en los cursos de capacitación, es culpar a los demás compañeros de trabajo y especialmente a los directivos por los malos procesos, es decir, muchos trabajadores tienden a victimizarse y a escudarse en la imposibilidad de hacer cambios, es por ello que, en esta actividad, se les pidió que enlistaran lo que no estaba en sus manos resolver, lo que ayudó a los trabajadores a desahogar sus preocupaciones, y a coincidir con sus compañeros.

Pero lo más importante por destacar: se dieron cuenta que así como había una lista de cosas que no podían resolver, como por ejemplo los horarios complicados, el estrés laboral, la actitud grosera o prepotente de algunos usuarios, entre otros; también había una gran lista de pequeñas cosas que sí podían hacer y que impactarían positivamente en la percepción del paciente, como por ejemplo, dar la bienvenida saludando, ver a los ojos, explicar con paciencia, interesarse por sus problemas de servicio, etc.

En la tercera etapa de decálogo de acciones específicas, se les presentaron a los participantes los 10 puntos trascendentales para atender los requerimientos del paciente, gestionar los mismos y obtener su satisfacción.

Puede observarse en las figuras 2 y 3 , los resultados de la encuesta de satisfacción que se entregó al participante al finalizar su curso. Se muestra un alto grado de aceptación ante la metodología de capacitación.

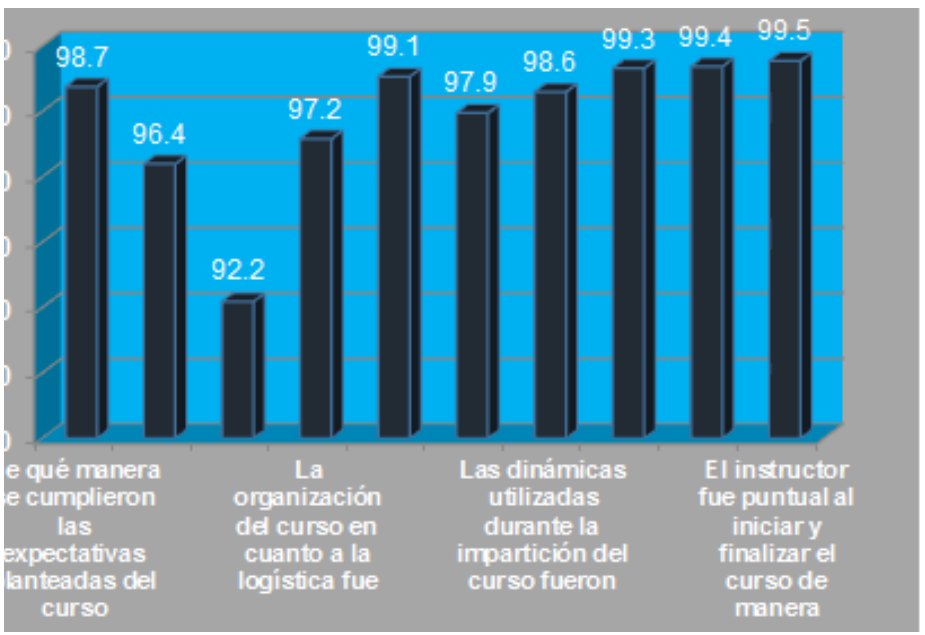

Figura 3. Gráfica del proceso por rubros de evaluación.

Fuente: Encuestas aplicadas en la capacitación

Como se mencionó con anterioridad, la etapa de evaluación de la efectividad de la capacitación, sobre la satisfacción del paciente, aun no finaliza, se encuentra en proceso. 


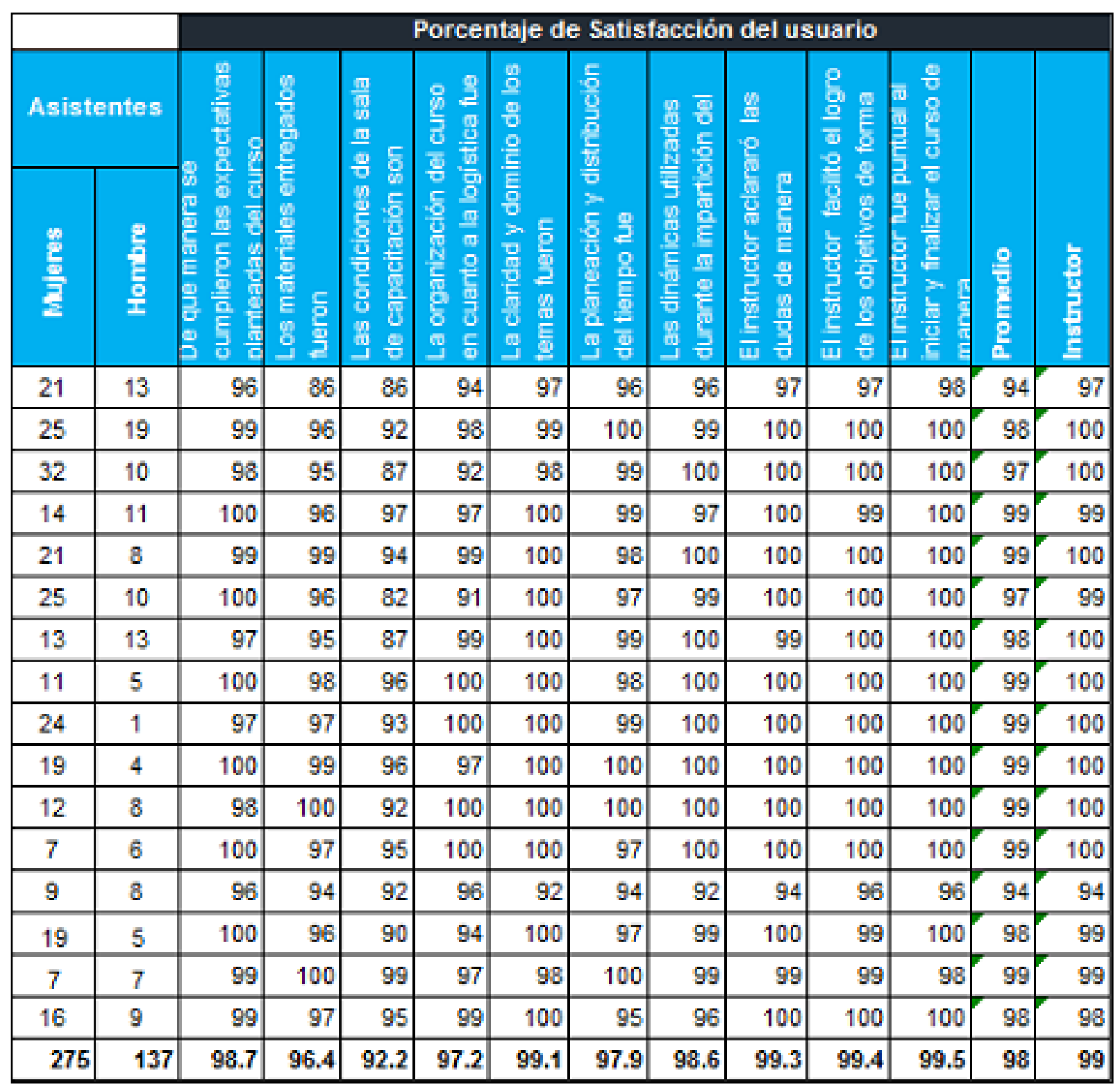

Figura 2. Porcentaje de satisfacción por sesión y tipo de usuario Fuente: elaboración propia

Propuesta metodológica. Por los resultados mencionados, es de vital importancia aplicar una metodología de aprendizaje significativo en el proceso de capacitación, lo que permitirá al capacitador realizar un trabajo adaptado al entorno del usuario.
La propuesta para el diseño e impartición se observa en la figura 4. 


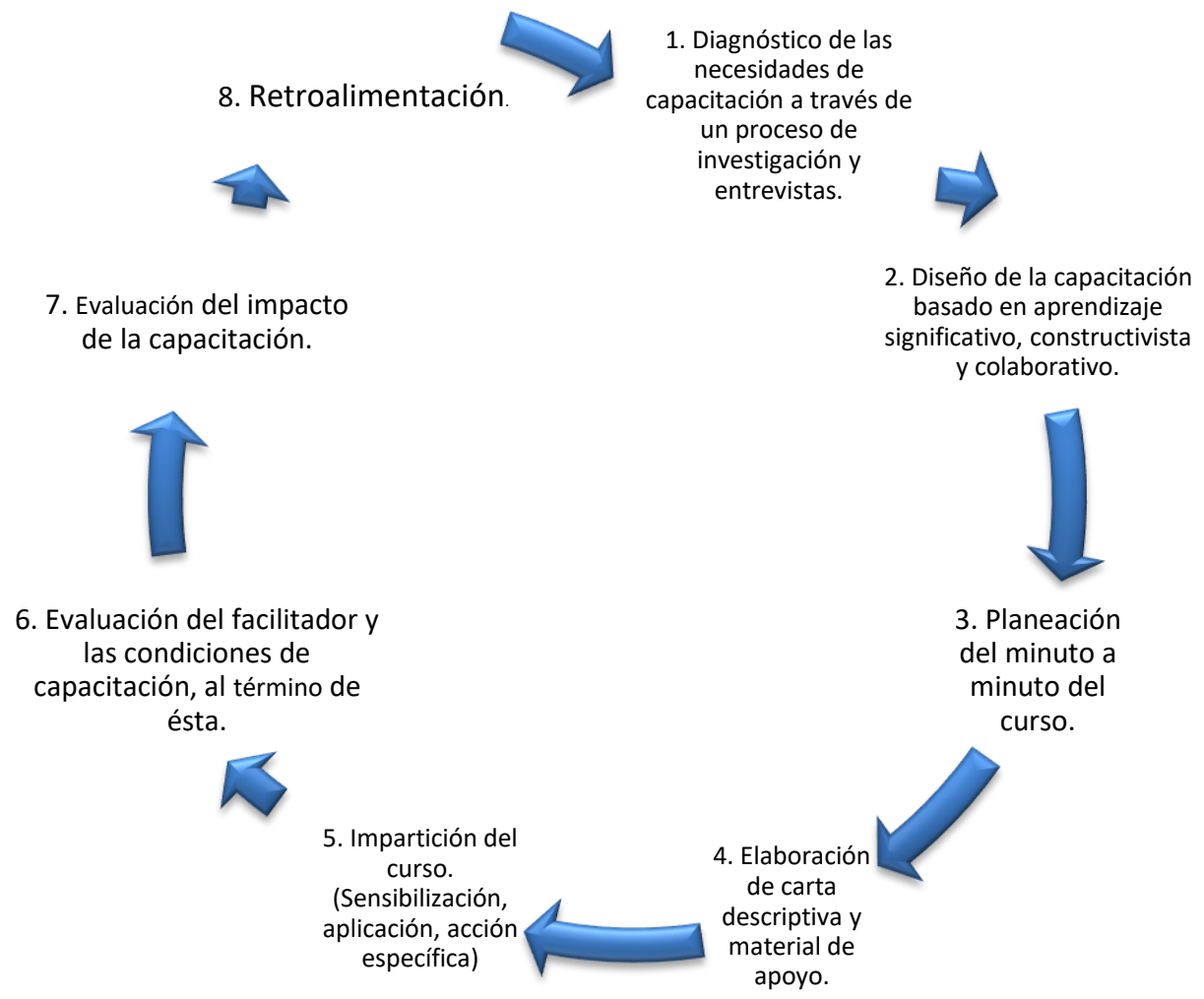

Figura 4. Modelo de Diseño de cursos y talleres para Empresas de Servicios Fuente: Elaboración propia

\section{Conclusiones}

La formación y desarrollo del capital humano en las organizaciones del sector servicios, proporciona a los empresarios un incremento de la productividad y mejoramiento de su calidad.

Aumenta la perspectiva estratégica además de la participación de su capital humano en el mercado, el establecimiento de nuevos mercados y el aumento de la versatilidad del empleado.

Genera satisfacción del cliente, que resulta en la disminución de las quejas, el ajuste de los tiempos de entrega/atención y la percepción de la imagen de la empresa.

Mejora los procesos internos, que surgen de la cohesión como grupo, disminuye la supervisión directa, y propicia el establecimiento de objetivos realistas y tangibles en las distintas áreas.

Permite mejorar su adaptabilidad al cambio.

Evita rotación de personal y consigue retener los talentos.

Motiva cuando no es impositiva, sino participativa.

Garantiza mejor desempeño y competitividad organizacional e individual.

El sector servicios, presenta características especiales, como son intangibilidad, subjetividad en las percepciones, heterogeneidad en la atención al usuario, etc.; por tanto, los retos de capacitación efectiva son muy 
altos. Lo que se pretende generar con esta metodología propuesta es una mejora en la competitividad del trabajador de este sector.

Para ello, se propone una metodología de diseño e impartición, basada en los estándares 217 y 301 de la norma CONOCER, pero adaptadas a las necesidades y particularidades del sector.

La metodología de 8 pasos propuesta se utilizó en el diseño e impartición de una capacitación al sector salud (IMSS), en la cual 412 personas fueron capacitadas y se obtuvieron resultados exitosos con respecto a la satisfacción de los participantes en el desempeño del capacitador.

Como puede observarse, los nuevos retos administrativos del siglo XXI, obligan a indagar e implementar nuevas estrategias de capacitación para impactar en el desempeño y la competitividad laboral que exigen cada vez más los clientes.

\section{Referencias}

Deloitte Consulting LLP y Bersin por Deloitte. (2014). Tendencias globales del capital humano 2014. Comprometiendo a la fuerza laboral del siglo XXI. Reino Unido: Deloitte University Press.

Arias, F., \& Heredia, V. (2006). Administración de Recursos Humanos para el alto desempeño. México: Trillas.

Constitución Política de los Estados Unidos Mexicanos. (2017). México: Berbera Editores.
Definición mx. (04 de Agosto de 2016). Definiciones, capital humano. Obtenido de Vía Definición.mx:http://definicion.mx/capitalhumano/

El Financiero. (21 de agosto de 2015). El Financiero.com.mx. Obtenido de http://www.elfinanciero.com.mx/economia/secto r-servicios-principal-motor-del-crecimientoeconomico.html

INEGI. (28 de Julio de 2015). cuentame.inegi.org.mx. parque servicios. Obtenido de http://cuentame.inegi.org.mx/economia/parque/s ervicios/i_servicios.pdf

Ley Federal del Trabajo. (2016). México: Berbera Editores.

Macías, M. (03 de Agosto de 2016). Guía de capacitación. La capacitación en la Empresa del siglo XXI. Obtenido de $\mathrm{http} /$ /www.guiadecapacitacion.com/ArticulosDestacados/la-capcitacion-en-la-empresa-delsiglo-xxi.htm1

OCDE. (ENERO de 2015). Estudios económicos de la OCDE México. Obtenido de http://www.oecd.org/economy/surveys/MexicoOverview-2015\%20Spanish.pdf

Pérez Paredes, A., Torralba Flores, A., \& D., M. Á. (2016). Fracaso Emprendedor, como experiencia de aprendizaje para empresarios. GEON, 4 (2).

Rodríguez, J., \& Morales, S. (2008). La capacitación en las organizaciones modernas. Synthesis. Punto de vista, 2.

Ruiz, X. (2016 de Agosto de 2016). Blog Grandes Pymes. Obtenido de http://www.grandespymes.com.ar/2012/11/11/im portancia-de-la-capacitacion-como-herramientadel-siglo-xxi/ 